\title{
Spatial pump-meter quantum correlations in a vectorial Kerr-medium model
}

\author{
Miguel Hoyuelos, ${ }^{1}$ Alice Sinatra, ${ }^{2}$ Pere Colet, ${ }^{1}$ Luigi Lugiato, ${ }^{2}$ and Maxi San Miguel ${ }^{1}$ \\ ${ }^{1}$ Instituto Mediterráneo de Estudios Avanzados IMEDEA* (CSIC-UIB), Campus Universitat Illes Balears, \\ E-07071 Palma de Mallorca, Spain \\ ${ }^{2}$ Istituto Nazionale della Materia, Dipartimento di Fisica dell'Universitá di Milano, Via Celoria 16, 20133 Milano, Italy
}

(Received 24 September 1998)

\begin{abstract}
We consider a vectorial Kerr-medium model including transverse spatial effects. We analyze cases in which, immediately above the threshold of the spatial instability, the homogeneous pump wave gives rise to two tilted waves corresponding to a stripe pattern in the near field. We analyze both the self-focusing and the selfdefocusing case and we point out the existence of anti-correlations between the quantum fluctuations of the intensity of the pump and the sum of the intensities of the two tilted waves creating the transverse pattern in the near field. We also evaluate the efficiency of this scheme as a quantum nondemolition (QND) scheme which uses the tilted waves as a "meter" to measure the intensity fluctuations of the pump. Our results show the posibility of a QND measurement in the self-defocusing case. In this case, and for a linearly polarized pump, the output pump beam (uniform in the transverse plane) and the pattern have orthogonal polarization and could be easily separated experimentally. [S1050-2947(99)06402-1]
\end{abstract}

PACS number(s): 42.50.Dv, 42.65.Sf, 42.50.Ct

\section{INTRODUCTION}

It is well known that transverse optical patterns [1,2] are capable of allowing noteworthy aspects linked to quantum fluctuations [2,3]. These issues have been mainly studied in a model of a cavity filled with Kerr medium and driven by a plane wave input field [3-5], in degenerate optical parametric oscillators [6-17], and in cavityless configurations for $\chi^{(2)}[15,18-23]$ and $\chi^{(3)}[24,25]$ media. The results of most of these papers are related to phenomena of quantum noise reduction or squeezing and to spatial quantum correlations; few of them discuss Einstein-Podolsky-Rosen aspects $[13,16]$. In the case of cavity systems, quantum phenomena arise both above $[3,4,10,16]$ and below $[6-9,11-15]$ the threshold for spontaneous pattern formation.

The original Kerr-medium model, formulated in [26] for a scalar electric field, has been generalized to include the vectorial character of the field [27]. Assuming an $x$-polarized input field, the generalized model displays a richer scenario of pattern forming instabilities that we describe below.

In the self-focusing case the system develops an instability that is formally identical to that of the scalar model [26]. In the case of two transverse dimensions it leads to the formation of a hexagonal pattern [4]; while in the case of one transverse dimension, as can be obtained by imposing a waveguide configuration, it leads to the formation of a stripe pattern in the near field. In the far field, this stripe pattern corresponds to a three-spot structure $[1,2]$, in which the central spot arises from the axial pump beam $(P)$, while the other two spots arise from two beams $\left(M_{1}\right.$ and $\left.M_{2}\right)$ generated by the spatial instability and propagating symmetrically with respect to the axis of the system (Fig. 1).

In the self-defocusing case, on the other hand, the system develops an instability, absent in the scalar model [26], lead-

*URL: http://www.imedea.uib.es/PhysDept/ ing to the formation of two $y$-polarized beams $M_{1}$ and $M_{2}$ $[27,28]$. In one or two transverse dimensions, the picture still corresponds to that of Fig. 1, in this case, however, the output pump beam $P_{\text {out }}$ is $x$ polarized, while the beams $M_{1}$ and $M_{2}$ are $y$ polarized. Hence in the near field the configuration of the output field (of frequency $\omega_{0}$ as the input field) is such that the $y$-polarized component corresponds to a stripe pattern, whereas the $\hat{x}$-polarized component is uniform in the transverse plane exactly as the input field.

In general pattern formation processes, the spatial instability is associated with a definite "critical" wave number $k_{c}$, which characterizes the periodicity of the pattern that arises immediately above the instability threshold [1,2]. Precisely, this pattern is given by a linear combination of plane waves $\exp (i \vec{k} \cdot \vec{r})[\vec{r} \equiv(x, y)$ is the position vector in the transverse plane, and $\vec{k} \equiv\left(k_{x}, k_{y}\right)$ is the wave vector], where $\vec{k}$ belongs to the critical circle $|\vec{k}|=k_{c}$. The selection of a dis-

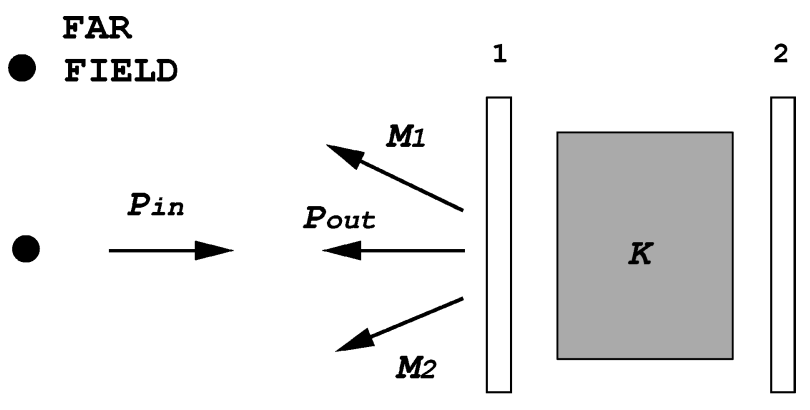

FIG. 1. Cavity with plane mirrors 1 and 2 contains the Kerr medium $K . \quad P_{\text {in }}$ is the monochromatic input pump field, which has a plane-wave configuration and frequency $\omega_{0}$. The input/output mirror 1 has a high reflectivity, while mirror 2 is completely reflecting. In addition to the output pump beam $P_{\text {out }}$, the spatial instability generates the two off-axial meter beams $M_{1}$ and $M_{2}$ of frequency $\omega_{0}$. Hence, the far field has a three-spot structure. 
crete set of wave vectors within this ring is a nonlinear process in which the correlations among the wave vectors play the crucial role. In the case of optical systems, the nonlinear process corresponds to the simultaneous absorption and emission of a number of photons, which gives rise to correlations of quantum nature. This is the very origin of the quantum aspects that characterize nonlinear optical patterns against spatial patterns in other fields.

In most of the analysis of quantum effects in nonlinear optical patterns in the configuration shown in Fig. 1, the interesting effects arise from the correlations between the two beams $M_{1}$ and $M_{2}$. In this paper, instead, we study the correlation between the pump beam and the pattern beams which turn out to be anticorrelated as previously shown within a classical framework [29]. This anticorrelation arises from the fact that in each elementary nonlinear process in Fig. 1 two photons of the pump beam are converted in one photon of beam $M_{1}$ and one photon of beam $M_{2}$. We perform this analysis in the framework of the vectorial Kerrmedium model, above the threshold of the spatial instability, both in the self-focusing case for one transverse dimension and in the self-defocusing case for one or two transverse dimensions. Immediately above threshold, we can use a simplified quantum model, in which the pump beam as well as beams $M_{1}$ and $M_{2}$ are described by single plane waves, of wave number 0 for the pump and wave number $k_{c}$ for the waves $M_{1}$ and $M_{2}$.

We analyze the anticorrelation using the concepts of quantum nondemolition (QND) measurements [30]. Precisely, we consider the beams $M_{1}$ and $M_{2}$ as meter beams to measure the quantum fluctuations of the pump beam that we will also call the signal beam. By thinking of the QND device as a "black box" with incoming and outgoing fields, QND measurements are characterized by three correlation coefficients: $C_{s}, C_{m}$, and $V_{s \mid m}$ relating the quantum fluctuations of the fields that enter or exit the black box [30,31].

The first coefficient $C_{s}$ measures the correlation between the incoming and the outgoing signal field that is the pump beam in our case. Such correlation is complete only for a perfectly nondestroying measurement for which $C_{s}=1$.

The second coefficient $C_{m}$ measures the correlation between the incoming signal and the outgoing meter, represented in our case by the two tilted waves $M_{1}$ and $M_{2}$ of Fig. 1. For $C_{m}=1$ the correlation is complete, so that by performing a direct measurement on the meter we perform an ideally accurate measurement of the incoming signal's fluctuations.

Finally the third coefficient $V_{s \mid m}$ quantifies the correlation between the outgoing signal and the outgoing meter. More precisely, due to its definition, $V_{s \mid m}$ represents the residual quantum noise in the outgoing signal once all the noise correlated to the outgoing meter has been subtracted; so that for a perfect correlation between the outgoing meter and outgoing signal one has $V_{s \mid m}=0$. In our case we study the conditional variance which links the intensity of the pump wave and the sum of the intensities of the two meter waves $M_{1}$ and $M_{2}$. The quantum nature of the correlation becomes manifest when the conditional variance becomes smaller than unity; once again this means that by measuring the intensity fluctuations of the meter beams $M_{1}$ and $M_{2}$ and by introducing an appropriate feedback loop, one can reduce the fluctuations of the pump beam below the shot-noise level
[30,31]. The complete QND regime is reached when, in addition to $V_{s \mid m}<1$, one has $C_{s}+C_{m}>1[30,31]$.

The outline of this paper is as follows. In Sec. II we derive the quantum-mechanical model for a Kerr medium with the polarization degree of freedom. In Sec. III we calculate the correlations between the quantum fluctuations of the different modes. The analysis shown in Secs. II and III is general and describes both self-focusing and self-defocusing cases. In Sec. IV we discuss our results for both cases. Finally, in Sec. V we summarize the work presented here and give some concluding remarks.

\section{VECTORIAL KERR-MEDIUM MODEL}

In this section we derive the quantum-mechanical counterpart of a semiclassical model [27-29] which accounts for the polarization degree of freedom of the electric field in an optical cavity filled with an isotropic $\chi^{(3)}$ nonlinear medium. The semiclassical equations that describe the behavior of the electric field $\vec{E}$ inside the cavity are

$$
\begin{aligned}
\frac{1}{\kappa} \frac{\partial E_{ \pm}}{\partial t}= & -\left(1+i \eta \theta_{0}\right) E_{ \pm}+i a \nabla^{2} E_{ \pm}+E_{0 \pm} \\
& +i \eta\left[\alpha\left|E_{ \pm}\right|^{2}+\beta\left|E_{\mp}\right|^{2}\right] E_{ \pm},
\end{aligned}
$$

where $E_{+}\left(E_{-}\right)$is the circularly right (left) polarized component of the field, $E_{0 \pm}$ are the components of the input field, $\eta$ takes the value $1(-1)$ for the self-focusing (selfdefocusing) case, $\eta \theta_{0}$ is the cavity detuning, $a$ is the strength of diffraction, $\nabla^{2}$ is the transverse Laplacian, $\kappa$ is the cavity decay rate, and $\alpha$ and $\beta$ [32] are parameters associated with the nonlinear susceptibility tensor $\chi^{(3)}$. Since we are considering an isotropic medium, $\alpha+\beta=2$ [33]. The scalar case described in $[3,26]$ can be recovered from Eq. (1) taking $E_{+}=E_{-}$, and rescaling the electrical field amplitude.

\section{A. Stability analysis of the homogeneous solution}

The steady-state homogeneous solutions of Eq. (1) are reference states from which transverse patterns emerge as they become unstable. We will consider an $x$ linearly polarized input field, i.e., $E_{0+}=E_{0-}=E_{0}$. In this case the homogeneous solution is also $x$ polarized, with $E_{s+}=E_{s-}=E_{s}$, and is given by the implicit equation

$$
I_{p}=I_{s}\left[1+\left(I_{s}-\theta_{0}\right)^{2}\right],
$$

where $I_{p}=2\left|E_{0}\right|^{2}$ and $I_{s}=2\left|E_{s}\right|^{2}$. As is well known Eq. (2) implies bistability for $\theta_{0}>\sqrt{3}$.

Basic features of the stability of the steady-state homogeneous solutions can be analyzed by considering the evolution equations for perturbations $\psi_{ \pm}$defined by

$$
E_{ \pm}=E_{s}\left[1+\psi_{ \pm}\right]
$$

From Eqs. (1) and (3) the linearized equations become

$$
\begin{aligned}
\partial_{t}^{\prime} \psi_{ \pm}= & -\left[1+i \eta\left(\theta_{0}-I_{s}\right)-i a \nabla^{2}\right] \psi_{ \pm} \\
& +i \eta I_{s}\left[\alpha\left(\psi_{ \pm}+\psi_{ \pm}^{*}\right)+(2-\alpha)\left(\psi_{\mp}+\psi_{\mp}^{*}\right)\right] / 2,
\end{aligned}
$$

where $t^{\prime}=\kappa t$ is the dimensionless time. 
It is convenient to make a change of variables to the following basis $[27,28]$ :

$$
\Sigma=\left\{\begin{array}{c}
\sigma_{1} \\
\sigma_{2} \\
\sigma_{3} \\
\sigma_{4}
\end{array}\right\}=\left\{\begin{array}{c}
\operatorname{Re}\left(\psi_{+}+\psi_{-}\right) \\
\operatorname{Im}\left(\psi_{+}+\psi_{-}\right) \\
\operatorname{Re}\left(\psi_{+}-\psi_{-}\right) \\
\operatorname{Im}\left(\psi_{+}-\psi_{-}\right)
\end{array}\right\} .
$$

In this basis, which emphasizes the role of symmetric $\left(\psi_{+}\right.$ $\left.=\psi_{-}\right)$and antisymmetric $\left(\psi_{+}=-\psi_{-}\right)$modes, Eq. (4) may be written as

$$
\partial_{t^{\prime}} \Sigma=L \Sigma
$$

where the linear matrix (in Fourier space) is

$$
\left.\begin{array}{cc}
0 & 0 \\
0 & 0 \\
-1 & -\eta\left(I_{s}-\theta_{k}\right) \\
\eta\left(I_{s}(2 \alpha-1)-\theta_{k}\right) & -1
\end{array}\right) \text {, }
$$

with

$$
\theta_{k}=\theta_{0}+\eta a k^{2} .
$$

$L$ is a matrix with $2 \times 2$ blocks in which the symmetric and antisymmetric modes are decoupled. As a consequence the linear instabilities lead to the growth of either a symmetric or an antisymmetric mode. The eigenvalues $\lambda$ of $L$ are [27]

$$
\begin{gathered}
\lambda_{1,2}=-1 \pm \sqrt{\left(\theta_{k}-3 I_{s}\right)\left(I_{s}-\theta_{k}\right)}, \\
\lambda_{3,4}=-1 \pm \sqrt{\left(\theta_{k}+(1-2 \alpha) I_{s}\right)\left(I_{s}-\theta_{k}\right)} .
\end{gathered}
$$

For the self-focusing case the homogeneous solution becomes unstable for $I_{s}^{c}=1$ with a critical wave number given by $a k_{c}^{2}=2-\theta_{0}$. The instability comes from the $\sigma_{1}, \sigma_{2}$ box of the linear matrix $L$ in Eq. (7). The critical mode is therefore symmetric and $x$ polarized.

In the self-defocusing case the homogeneous solution becomes unstable for $I_{s}^{c}=1 /(1-\alpha)$ and the critical wave number is given by $a k_{c}^{2}=\theta_{0}-\alpha /(1-\alpha)$. The instability comes from the $\sigma_{3}, \sigma_{4}$ box of $L$, so that the critical mode is an antisymmetric mode and $y$ polarized.

\section{B. Quantum formulation}

The approach that we follow to obtain the quantummechanical version of Eq. (1) is described in [3], where the scalar model was studied. We assume periodic boundary conditions in the transverse plane in a square of side $b$ for the self-defocusing case or a segment of length $b$ for the selffocusing case. The master equation for the density operator $\rho$ is

$$
\dot{\rho}=\sum_{\vec{n}} \Lambda_{\vec{n}+} \rho+\sum_{\vec{n}} \Lambda_{\vec{n}-} \rho-\frac{i}{\hbar}[H, \rho] .
$$

The dot designates derivatives with respect to the dimensionless time $t^{\prime}=\kappa t$. The Liouvillians $\Lambda_{\vec{n} \pm}$ are

$$
\Lambda_{\vec{n} \pm} \rho=\left[\hat{a}_{\vec{n} \pm} \rho, \hat{a}_{\vec{n} \pm}^{\dagger}\right]+\left[\hat{a}_{\vec{n} \pm}, \rho \hat{a}_{\vec{n} \pm}^{\dagger}\right]
$$

where $\hat{a}_{n+}^{\dagger}$ and $\hat{a}_{\vec{n}+}\left(\hat{a}_{n-}^{\dagger}\right.$ and $\left.\hat{a}_{\vec{n}-}\right)$ are the creation and annihilation operators of circularly right (left) polarized photons with wave vector $\vec{k}=(2 \pi / b) \vec{n}$, where $\vec{n}=\left(n_{x}\right)$ for the self-focusing case and $\vec{n}=\left(n_{x}, n_{y}\right)$ for the self-defocusing case, with $n_{x}, n_{y}=0, \pm 1, \pm 2, \ldots$.

The Hamiltonian is the sum of three parts $H=H_{0}+H_{\text {ext }}$ $+H_{\text {int }}$. The free Hamiltonian is given by

$$
H_{0}=\hbar \eta \sum_{\vec{n}} \theta_{n}\left(\hat{a}_{\vec{n}+}^{\dagger} \hat{a}_{\vec{n}+}+\hat{a}_{\vec{n}-}^{\dagger} \hat{a}_{\vec{n}-}\right) .
$$

The mode detuning $\eta \theta_{n}$ is given by $\eta \theta_{n}=\eta \theta_{0}$ $+4 \pi^{2} a n^{2} / b^{2}$ where $n \equiv|\vec{n}|$.

The external Hamiltonian representing the driving field is

$$
H_{\mathrm{ext}}=i \hbar \alpha_{\mathrm{I}+}\left(\hat{a}_{0+}^{\dagger}-\hat{a}_{0+}\right)+i \hbar \alpha_{\mathrm{I}-}\left(\hat{a}_{0-}^{\dagger}-\hat{a}_{0-}\right),
$$

where $\alpha_{\mathrm{I}^{ \pm}}=E_{0 \pm} / \sqrt{g}, g$ being the absolute value of the coupling constant, defined as $g=2 C /\left(|\Delta|^{3} N_{s}\right) . \quad C$ is the bistability parameter, $\Delta$ is the atomic detuning, and $N_{s}$ is the saturation parameter [3]. In our definition $g$ is always positive, the sign being denoted by $\eta$ which is +1 for selffocusing and -1 for self-defocusing. Finally, the modes represented by the operators $\hat{a}_{\vec{n} \pm}$ are coupled via the interaction Hamiltonian,

$$
\begin{aligned}
H_{\text {int }}= & -\hbar \eta g b^{2} \iint d x d y \\
& \times\left(\frac{\alpha}{2} A_{+}^{\dagger}{ }^{2} A_{+}{ }^{2}+\beta A_{+}^{\dagger} A_{-}^{\dagger} A_{+} A_{-}+\frac{\alpha}{2} A_{-}^{\dagger}{ }^{2} A_{-}{ }^{2}\right),
\end{aligned}
$$

where $A_{ \pm}(x, y)$ is proportional to the field envelope and

$$
A_{ \pm}(x, y)=\frac{1}{b} \sum_{\vec{n}} \hat{a}_{\vec{n} \pm} \exp \left(i \vec{k}_{\vec{n}} \cdot \vec{r}\right)
$$

with $\vec{r} \equiv(x, y)$. Equation (1) can be recovered from the evolution equation for the operator $A_{ \pm}$, by taking $E_{ \pm} / \sqrt{g}$ 
$=\left\langle A_{ \pm}\right\rangle$and using the semiclassical approximation $\langle A B\rangle$ $=\langle A\rangle\langle B\rangle$, where $A$ and $B$ are quantum operators.

In this work we are going to consider only the case of linearly polarized input field and we restrict ourselves to the case $\theta_{0}<\sqrt{3}$, where the homogeneous solution is monostable. As described in the Introduction and in Sec. II A, the linear stability analysis of the semiclassical equations shows different scenarios in the self-focusing or in the self-defocusing case. In the first one, when the homogeneous solution becomes unstable, a hexagonal pattern is formed in systems with two transverse dimensions, while for systems with one relevant transverse dimension, a stripe pattern emerges. When the input is linearly $x$ polarized, the linear stability analysis shows that the pattern produced above threshold is also $x$ polarized. In the second case (selfdefocusing), a stripe pattern develops, and, if the input is $x$ polarized, the pattern is polarized in the $y$ direction.

In the two cases in which stripes are formed, the field can be described near threshold in terms of only three transverse modes for each polarization component of the field: the homogeneous one, and two with wave vectors $+\vec{k}_{c}$ and $-\vec{k}_{c}$. Labeling these three modes 0,1 , 2, we have

$$
A_{ \pm}=\frac{1}{b}\left(\hat{a}_{0 \pm}+\hat{a}_{1 \pm} e^{i \vec{k}_{c} \cdot \vec{r}}+\hat{a}_{2 \pm} e^{-i \vec{k}_{c} \cdot \vec{r}}\right) .
$$

Since we will consider a linearly polarized input, for definiteness along the $x$ direction, it is convenient to use a base where the modes are linearly polarized. We introduce the following change of variables: $\hat{a}_{i}=\left(\hat{a}_{i+}+\hat{a}_{i-}\right) / \sqrt{2}$ ( $x$ polarized) and $\hat{b}_{i}=\left(\hat{a}_{i+}-\hat{a}_{i-}\right) / \sqrt{2}$ ( $y$ polarized $)$. On this basis, the fields $A_{+}$and $A_{-}$are

$$
A_{ \pm}=\frac{1}{\sqrt{2} b}\left[\hat{a}_{0} \pm \hat{b}_{0}+\left(\hat{a}_{1} \pm \hat{b}_{1}\right) e^{i \vec{k}_{c} \cdot \vec{r}}+\left(\hat{a}_{2} \pm \hat{b}_{2}\right) e^{-i \vec{k}_{c} \cdot \vec{r}}\right]
$$

The free Hamiltonian becomes

$$
\begin{aligned}
H_{0}= & \hbar \eta\left[\theta_{0}\left(\hat{a}_{0}^{\dagger} \hat{a}_{0}+\hat{b}_{0}^{\dagger} \hat{b}_{0}\right)\right. \\
& \left.+\theta_{1}\left(\hat{a}_{1}^{\dagger} \hat{a}_{1}+\hat{a}_{2}^{\dagger} \hat{a}_{2}+\hat{b}_{1}^{\dagger} \hat{b}_{1}+\hat{b}_{2}^{\dagger} \hat{b}_{2}\right)\right],
\end{aligned}
$$

with

$$
\theta_{1}=\theta_{0}+\eta a\left|\vec{k}_{c}\right|^{2}
$$

The external Hamiltonian is

$$
H_{\mathrm{ext}}=i \hbar \alpha_{I}\left(\hat{a}_{0}^{\dagger}-\hat{a}_{0}\right),
$$

where, since the input field is $x$ polarized, $\alpha_{I} / \sqrt{2}=\alpha_{I+}$ $=\alpha_{I-}$ and, for definiteness, $\alpha_{I}$ is assumed real. From the definition of $\alpha_{I \pm}$ and the definition of $I_{p}$ given after Eq. (2), we have $\alpha_{I}^{2}=I_{p} / g$.

The interaction Hamiltonian can be written as $H_{\text {int }}$ $=H_{\mathrm{FWM}}+H_{\mathrm{CPM}}+H_{\mathrm{SPM}}$, where each part corresponds to the following microscopic processes: four wave mixing,

$$
\begin{aligned}
H_{\mathrm{FWM}}= & -\hbar g \eta\left[\hat{a}_{0}^{2} \hat{a}_{1}^{\dagger} \hat{a}_{2}^{\dagger}+\hat{b}_{0}^{2} \hat{b}_{1}^{\dagger} \hat{b}_{2}^{\dagger}\right. \\
& +(\alpha-1)\left(\hat{a}_{0}^{2} \hat{b}_{1}^{\dagger} \hat{b}_{2}^{\dagger}+\hat{b}_{0}^{2} \hat{a}_{1}^{\dagger} \hat{a}_{2}^{\dagger}\right) \\
& +\alpha \hat{a}_{0} \hat{b}_{0}\left(\hat{a}_{1}^{\dagger} \hat{b}_{2}^{\dagger}+\hat{a}_{2}^{\dagger} \hat{b}_{1}^{\dagger}\right) \\
& +\sum_{i<j}\left[2(\alpha-1) \hat{a}_{i} \hat{a}_{j} \hat{b}_{i}^{\dagger} \hat{b}_{j}^{\dagger}+\alpha \hat{a}_{j}^{\dagger} \hat{b}_{i}^{\dagger} \hat{a}_{i} \hat{b}_{j}\right] \\
& \left.+\frac{1}{2}(\alpha-1) \sum_{i} \hat{a}_{i}^{\dagger} \hat{b}_{i}^{2}\right]+ \text { H.c. },
\end{aligned}
$$

cross phase modulation,

$$
\begin{aligned}
H_{\mathrm{CPM}}= & -\hbar g \eta \\
& \times\left[2 \sum_{i<j}\left(\hat{a}_{i}^{\dagger} \hat{a}_{i} \hat{a}_{j}^{\dagger} \hat{a}_{j}+\hat{b}_{i}^{\dagger} \hat{b}_{i} \hat{b}_{j}^{\dagger} \hat{b}_{j}\right)+\alpha \sum_{i, j} \hat{a}_{i}^{\dagger} \hat{b}_{j}^{\dagger} \hat{a}_{i} \hat{b}_{j}\right],
\end{aligned}
$$

and self-phase modulation,

$$
H_{\mathrm{SPM}}=-\frac{\hbar g \eta}{2} \sum_{i}\left(\hat{a}_{i}^{\dagger 2} \hat{a}_{i}^{2}+\hat{b}_{i}^{\dagger 2} \hat{b}_{i}^{2}\right)
$$

where we have used the relation $\beta=2-\alpha$.

In the self-focusing case $(\eta=1)$, if we set $\hat{b}_{0}=\hat{b}_{1}=\hat{b}_{2}$ $=0$, these Hamiltonians are independent of $\alpha$ and become identical to those of the scalar case $[3,34]$.

\section{QUANTUM FLUCTUATIONS}

We are interested in the quantum correlations between the intensity fluctuations of the pump mode $\hat{a}_{0}$ and the fluctuations in the sum of the intensities of the two transverse modes $\hat{a}_{1}$ and $\hat{a}_{2}$ (self-focusing) or $\hat{b}_{1}$ and $\hat{b}_{2}$ (selfdefocusing). Due to the structure of the Hamiltonian, we expect to find strong correlations between these variables near the threshold for the pattern formation.

The time evolution equations in the semiclassical approximation are obtained by factoring mean values of products into products of mean values. In the following we indicate by $a_{i}, b_{i}(i=0,1,2)$ the mean values $\left\langle\hat{a}_{i}\right\rangle$ and $\left\langle\hat{b}_{i}\right\rangle$ of the corresponding operators $\left(a_{i}^{*}, b_{i}^{*}\right.$ are their complex conjugates).

For the self-focusing case we look for stationary solutions polarized in the $x$ direction, $b_{i}^{s}=0$. From the evolution equations, for the stationary solution, we obtain

$$
\begin{gathered}
0=\left[2 a_{0}^{*} a_{1} a_{2}+a_{0}^{*} a_{0}^{2}+2 a_{0}\left(a_{2}^{*} a_{2}+a_{1}^{*} a_{1}\right)\right] i g \\
-\left(1+i \theta_{0}\right) a_{0}+\alpha_{I}, \\
0=\left[a_{2}^{*} a_{0}^{2}+a_{1}^{*} a_{1}^{2}+2 a_{1}\left(a_{2}^{*} a_{2}+a_{0}^{*} a_{0}\right)\right] i g-\left(1+i \theta_{1}\right) a_{1} . \\
0=\left[a_{1}^{*} a_{0}^{2}+a_{2}^{*} a_{2}^{2}+2 a_{2}\left(a_{1}^{*} a_{1}+a_{0}^{*} a_{0}\right)\right] i g-\left(1+i \theta_{1}\right) a_{2} .
\end{gathered}
$$


Here we consider solutions such that $a_{i}^{s}=\left|a_{i}^{s}\right| e^{i \phi_{i}}$, with $\left|a_{1}^{s}\right|=\left|a_{2}^{s}\right|$, and we use, for the sake of brevity, the following notation for the intensities of the homogeneous and meter modes: $I_{0}=g\left|a_{0}^{s}\right|^{2}$ and $I_{1}=g\left|a_{1}^{s}\right|^{2}=g\left|a_{2}^{s}\right|^{2}$. In this case, Eqs. (25) and (26) yield

$$
e^{i \phi}=\frac{1}{I_{0}}\left[\theta_{1}-3 I_{1}-2 I_{0}-i\right],
$$

where $\phi=2 \phi_{0}-\phi_{1}-\phi_{2}$. Taking the modulus squared of Eq. (27) we derive an expression for $I_{0}$ as a function of $I_{1}$,

$$
I_{0}=\frac{2\left(\theta_{1}-3 I_{1}\right)-\sqrt{\left(\theta_{1}-3 I_{1}\right)^{2}-3}}{3} .
$$

Using the relation (27) in Eq. (24) we find for the pump intensity $\alpha_{I}^{2}$ and the phase $\phi_{0}$

$$
\begin{gathered}
I_{p}=g \alpha_{I}^{2}=I_{0}\left[\left(\theta_{0}-I_{0}-2 \frac{I_{1}}{I_{0}}\left(\theta_{1}-3 I_{1}\right)\right)^{2}+\left(1+2 \frac{I_{1}}{I_{0}}\right)^{2}\right], \\
e^{i \phi_{0}}=\frac{\sqrt{I_{p}}}{\sqrt{I_{0}}\left[i \eta\left(\theta_{0}-I_{0}-2\left(I_{1} / I_{0}\right)\left(\theta_{1}-3 I_{1}\right)\right)+1+2 I_{1} / I_{0}\right]} .
\end{gathered}
$$

Finally, the amplitudes of the stationary solution $I_{1}$ and $I_{0}$ are obtained solving simultaneously the implicit equations (28) and (29) for a given pump $\alpha_{I}$. The phases $\phi_{0}$ and $\phi$ are given by Eqs. (30) and (27), respectively. Note that this only fixes the value of the sum $\phi_{1}+\phi_{2}$, not the individual values $\phi_{1}, \phi_{2}$.

In order to deal with intensity and phase fluctuations, it is convenient to introduce the variables $\bar{a}_{i}=a_{i} \exp \left(-i \phi_{i}\right)(i$ $=0,1,2)$, where $\phi_{i}$ are the stationary values of the phases $\left(\phi_{1}\right.$ and $\phi_{2}$ are chosen arbitrarily with the link that $\phi_{1}$ $+\phi_{2}$ has the correct value). For simplicity, we drop the bar in the rest of the paper. We now separate the mean value and the fluctuations as

$$
a_{i}(t)=a_{i}^{s}+\delta a_{i}=\sqrt{I_{i} / g}+\delta a_{i}
$$

for $i=0,1,2$ and $I_{2}=I_{1}$. Linearizing with respect to the fluctuations, one sees that the terms containing $b_{i}$ and $b_{i}^{*}$ ( $i$ $=0,1,2)$ do not contribute in the linearized approximation (because $b_{i}^{s}=0$ ) and the equations for the fluctuations $\delta a_{i}$ read

$$
\begin{aligned}
\delta \dot{a}_{0}= & -\left(1+i \theta_{0}\right) \delta a_{0}+2 i\left(I_{0}+2 I_{1}\right) \delta a_{0} \\
& +i\left(I_{0}+2 I_{1} e^{-i \phi}\right) \delta a_{0}^{*} \\
& +2 i \sqrt{I_{0} I_{1}}\left(1+e^{-i \phi}\right)\left(\delta a_{1}+\delta a_{2}\right) \\
& +2 i \sqrt{I_{0} I_{1}}\left(\delta a_{1}^{*}+\delta a_{2}^{*}\right) \\
\delta \dot{a}_{1}= & -\left(1+i \theta_{1}\right) \delta a_{1}+2 i\left(2 I_{1}+I_{0}\right) \delta a_{1}+i I_{1} \delta a_{1}^{*} \\
+ & 2 i \sqrt{I_{0} I_{1}}\left(1+e^{i \phi}\right) \delta a_{0}+2 i \sqrt{I_{0} I_{1}} \delta a_{0}^{*}+2 i I_{1} \delta a_{2} \\
+ & i\left(2 I_{1}+I_{0} e^{i \phi}\right) \delta a_{2}^{*},
\end{aligned}
$$

where we took into account that $g\left|a_{0}^{s}\right|=\sqrt{I_{0}}, g\left|a_{1}^{s}\right|=g\left|a_{2}^{s}\right|$ $=\sqrt{I_{1}}$. The equation for $\delta \dot{a}_{2}$ is obtained by exchanging the indexes 1 and 2 in Eq. (33).

For the self-defocusing case, since the meter is $y$ polarized, we look for stationary solutions such that $a_{0}^{s}$ $=\left|a_{0}^{s}\right| e^{i \phi_{0}}, \quad a_{1}^{s}=a_{2}^{s}=0, \quad b_{0}^{s}=0, \quad b_{1}^{s}=\left|b_{1}^{s}\right| e^{i \psi_{1}}, \quad$ and $\quad b_{2}^{s}$ $=\left|b_{2}^{s}\right| e^{i \psi_{2}}$, with $\left|b_{1}^{s}\right|=\left|b_{2}^{s}\right|$. We will use the same notation as before for the homogeneous and meter stationary intensities: $I_{0}=g\left|a_{0}^{s}\right|^{2}$ and $I_{1}=g\left|b_{1}^{s}\right|^{2}$. The stationary solution is obtained as in the self-focusing case and Eqs. (29) and (30) are the same. Equations (27) and (28) now become

$$
\begin{gathered}
e^{i \phi}=\frac{1}{(\alpha-1) I_{0}}\left[\theta_{1}-3 I_{1}-\alpha I_{0}+i\right], \\
I_{0}=\frac{\alpha\left(\theta_{1}-3 I_{1}\right)-\sqrt{(\alpha-1)^{2}\left(\theta_{1}-3 I_{1}\right)^{2}+1-2 \alpha}}{(2 \alpha-1)},
\end{gathered}
$$

where now $\phi$ is defined as $\phi=2 \phi_{0}-\psi_{1}-\psi_{2}$.

As we did before, we introduce the variables $\bar{a}_{0}$ $=a_{i} \exp \left(-i \phi_{0}\right), \bar{b}_{i}=b_{i} \exp \left(-i \psi_{i}\right)(i=1,2)$, and we drop the bar in the rest of the paper, for simplicity. We then set

$$
\begin{aligned}
& a_{0}(t)=\sqrt{I_{0} / g}+\delta a_{0}(t), \\
& b_{1}(t)=\sqrt{I_{1} / g}+\delta b_{1}(t), \\
& b_{2}(t)=\sqrt{I_{1} / g}+\delta b_{2}(t) .
\end{aligned}
$$

Linearizing with respect to the fluctuations, we obtain in this case a closed set of equations for $\delta a_{0}, \delta b_{1}$, and $\delta b_{2}$ which reads

$$
\begin{aligned}
\delta \dot{a}_{0}= & -\left(1-i \theta_{0}\right) \delta a_{0}-2 i\left(I_{0}+\alpha I_{1}\right) \delta a_{0} \\
& -i\left[I_{0}+2(\alpha-1) I_{1} e^{-i \phi}\right] \delta a_{0}^{*} \\
& -i\left[\alpha+2(\alpha-1) e^{-i \phi}\right] \sqrt{I_{0} I_{1}}\left(\delta a_{1}+\delta a_{2}\right) \\
& -i \alpha \sqrt{I_{0} I_{1}}\left(\delta a_{1}^{*}+\delta a_{2}^{*}\right), \\
\delta \dot{b}_{1}=- & \left(1-i \theta_{1}\right) \delta b_{1}-i\left(4 I_{1}+\alpha I_{0}\right) \delta b_{1}-i I_{1} \delta b_{1}^{*} \\
- & i\left[\alpha+2(\alpha-1) e^{i \phi}\right] \sqrt{I_{0} I_{1}} \delta a_{0}-i \alpha \sqrt{I_{0} I_{1}} \delta a_{0}^{*} \\
- & 2 i I_{1} \delta b_{2}-i\left[2 I_{1}+(\alpha-1) I_{0} e^{i \phi}\right] \delta b_{2}^{*} .
\end{aligned}
$$

The equation for $\delta \dot{b}_{2}$ is obtained exchanging the indexes 1 and 2 in Eq. (38).

We now define the quadratures of the pump mode and of the sum and difference of the transverse modes as

$$
\begin{gathered}
\delta X_{0}, \quad \delta Y_{0}, \\
\delta X_{+}=\frac{\delta X_{1}+\delta X_{2}}{\sqrt{2}}, \quad \delta Y_{+}=\frac{\delta Y_{1}+\delta Y_{2}}{\sqrt{2}}, \\
\delta X_{-}=\frac{\delta X_{1}-\delta X_{2}}{\sqrt{2}}, \quad \delta Y_{-}=\frac{\delta Y_{1}-\delta Y_{2}}{\sqrt{2}},
\end{gathered}
$$


where, for the self-focusing case, $\delta X_{j}=\delta a_{j}+\delta a_{j}^{*}$ and $\delta Y_{j}$ $=-i \delta a_{j}+i \delta a_{j}^{*}$ for $j=0,1,2$. For the self-defocusing case the meter modes are $y$ polarized and we have $\delta X_{j}=\delta b_{j}$ $+\delta b_{j}^{*}, \quad \delta Y_{j}=-i \delta b_{j}+i \delta b_{j}^{*}$, for $j=1,2$. The reason for the denominator $\sqrt{2}$ in Eqs. (39) is to have the shot noise of the new variables normalized to one.

Using the linearized equations, we calculate the drift matrix $A$ and the diffusion matrix $D$ for the classical-looking Fokker-Planck equation in the $P$ representation, using the indexes $1, \ldots, 6$ for the components of the vector $\vec{\gamma}$ $=\left(\delta a_{0}, \delta a_{0}^{*}, \delta a_{1}, \delta a_{1}^{*}, \delta a_{2}, \delta a_{2}^{*}\right)$ for the self-focusing case or $\quad \vec{\gamma}=\left(\delta a_{0}, \delta a_{0}^{*}, \delta b_{1}, \delta b_{1}^{*}, \delta b_{2}, \delta b_{2}^{*}\right)$ for the selfdefocusing case. The elements of matrices $A$ and $D$ are given in the Appendix.

It is then straightforward to calculate the spectral matrix $M[35]$ :

$$
M(\omega)=(A+i \omega I)^{-1} D\left(A^{T}-i \omega I\right)^{-1},
$$

and the response matrix $R[36]$ :

$$
R(\omega)=(A+i \omega I)^{-1} C
$$

where $C$ is the matrix of the equal time commutators $C_{i, j}$ $=\left\langle\left[\hat{\gamma}_{i}, \hat{\gamma}_{j}\right]\right\rangle$. From matrices $M$ and $R$ all quantum correlations can be obtained.

We are interested in the intensity fluctuations of the three relevant modes. In the linearized regime, instead of intensity fluctuations one can equivalently consider the fluctuations of the quadrature components which correspond to the amplitudes. Since there is no input (apart from vacuum noise) for modes 1 and 2, the output phase coincides with the intracavity phases $\phi_{1}$ and $\phi_{2}$ for these modes, so that the amplitude quadrature components are $X_{1}$ and $X_{2}$, in the sense that the fluctuations $\delta I_{i}$ are equal to $2 \sqrt{g I_{i}} \delta X_{i}\left(i=1,2, I_{1}=I_{2}\right)$. So, the fluctuations in the sum $I_{1}+I_{2}$ correspond to the noise in the quadrature component $X_{+}^{\text {out }}$, defined in the self-focusing case as $X_{+}^{\text {out }}=\left(a_{1}+a_{1}^{*}+a_{2}+a_{2}^{*}\right) / \sqrt{2}$ and in the selfdefocusing case as $X_{+}^{\text {out }}=\left(b_{1}+b_{1}^{*}+b_{2}+b_{2}^{*}\right) / \sqrt{2}$. For the pump mode 0 , instead, there is a nonvanishing input field and therefore the output phase is different from the intracavity phase $\phi_{0}$. The intensity fluctuations correspond to the noise in the quadrature component

$$
X_{0}^{\text {out }}=a_{0} e^{-i \Theta_{0}^{\text {out }}}+a_{0}^{\dagger} e^{i \Theta_{0}^{\text {out }}},
$$

where $\Theta_{0}^{\text {out }}$ is the output phase of the mode $a_{0}$. The calculation of the phase $\Theta_{0}^{\text {out }}$ is done using the input-output relation

$$
a_{0}^{\text {out }}=2 a_{0}-a_{0}^{\text {in }} .
$$

By definition $a_{0}=\sqrt{I_{0} / g}$ and $a_{0}^{\text {in }}=\alpha_{I} e^{-i \phi_{0}}=\sqrt{I_{p} / g} e^{-i \phi_{0}}$ with $\alpha_{I}$ real, we then obtain from Eqs. (30) and (43)

$$
e^{i \Theta_{0}^{\text {out }}}=\sqrt{\frac{I_{0}}{I_{0}^{\text {out }}}}\left[1-2 \frac{I_{1}}{I_{0}}-i \eta\left(\theta_{0}-I_{0}-2 \frac{I_{1}}{I_{0}}\left(\theta_{1}-3 I_{1}\right)\right)\right],
$$

where $I_{0}^{\text {out }}=g\left|a_{0}^{\text {out }}\right|^{2}$. Equation (44) is valid for the selffocusing and self-defocusing case.

The expressions for the squeezing spectra of the amplitudes $X_{0}^{\text {out }}$ and $X_{+}$are [36]

$$
\begin{aligned}
S_{X_{0}^{\text {out }}} & =\left\langle\delta X_{0}^{\text {out }} \delta X_{0}^{\text {out }}\right\rangle_{\omega} \\
& =1+2\left(M_{12}+M_{21}+M_{11} e^{-i 2 \Theta_{0}^{\text {out }}}+M_{22} e^{i 2 \Theta_{0}^{\text {out }}}\right),
\end{aligned}
$$

$$
\begin{aligned}
S_{X_{+}^{\text {out }}}= & \left\langle\delta X_{+}^{\text {out }} \delta X_{+}^{\text {out }}\right\rangle_{\omega}=1+M_{34}+M_{43}+M_{33}+M_{44}+M_{56} \\
& +M_{65}+M_{55}+M_{66}+M_{35}+M_{36}+M_{45}+M_{46}+M_{53} \\
& +M_{54}+M_{63}+M_{64} .
\end{aligned}
$$

The notation \langle\rangle$_{\omega}$ means Fourier transform of the symmetrized correlation, and it is defined, for some generic variables $W$ and $Z$, as

$$
\langle W Z\rangle_{\omega}=\int_{-\infty}^{\infty}\langle W(t) Z(0)\rangle_{\mathrm{symm}} e^{-i \omega t} d t
$$

We are interested in the conditional variance of $X_{0}$ given the result of a measurement on $X_{+}[31]$ :

$$
V_{s \mid m}\left[X_{0}^{\text {out }} \mid X_{+}^{\text {out }}\right]=S_{X_{0}^{\text {out }}}\left(1-\frac{\left|\left\langle\delta X_{0}^{\text {out }} \delta X_{+}^{\text {out }}\right\rangle_{\omega}\right|^{2}}{S_{X_{0}^{\text {out }}} S_{X_{+}^{\text {out }}}}\right),
$$

with

$$
\begin{aligned}
\left\langle\delta X_{0}^{\text {out }} \delta X_{+}^{\text {out }}\right\rangle_{\omega}= & -\sqrt{2}\left[\left(M_{31}+M_{41}+M_{51}+M_{61}\right) e^{-i \Theta_{0}^{\text {out }}}\right. \\
& \left.+\left(M_{32}+M_{42}+M_{52}+M_{62}\right) e^{i \Theta_{0}^{\text {out }}}\right]
\end{aligned}
$$

With these definitions, the shot noise is normalized to 1 . For a QND measurement one requires that the information gained by the measurement is sufficient to reduce the fluctuation of the signal beam (pumping) below the shot-noise level, corresponding to $V_{s \mid m}\left[X_{0}^{\text {out }} \mid X_{+}^{\text {out }}\right]<1$.

Additionally, we study how the fluctuations are transferred from the signal input to the signal output (the nondemolition character of the measurement), and from the signal input to the meter output or pattern modes (accuracy of the measurement). We consider the normalized correlations, first introduced in [31], defined as

$$
\begin{gathered}
C_{s}=\frac{\left|\left\langle\delta X_{0}^{\text {in }} \delta X_{0}^{\text {out }}\right\rangle_{\omega}\right|^{2}}{\left\langle\delta X_{0}^{\text {in }} \delta X_{0}^{\text {in }}\right\rangle_{\omega}\left\langle\delta X_{0}^{\text {out }} \delta X_{0}^{\text {out }}\right\rangle_{\omega}}, \\
C_{m}=\frac{\left|\left\langle\delta X_{0}^{\text {in }} \delta X_{+}^{\text {out }}\right\rangle_{\omega}\right|^{2}}{\left\langle\delta X_{0}^{\text {in }} \delta X_{0}^{\text {in }}\right\rangle_{\omega}\left\langle\delta X_{+}^{\text {out }} \delta X_{+}^{\text {out }}\right\rangle_{\omega}},
\end{gathered}
$$

where

$$
\begin{aligned}
\left\langle\delta X_{0}^{\text {in }} \delta X_{0}^{\text {out }}\right\rangle_{\omega}= & -\cos \left(\Theta_{0}^{\text {out }}-\Theta_{0}^{\text {in }}\right)-R_{11} e^{-i\left(\Theta_{0}^{\text {out }}+\Theta_{0}^{\text {in }}\right)} \\
& +R_{22} e^{i\left(\Theta_{0}^{\text {out }}+\Theta_{0}^{\text {in }}\right)}+R_{12} e^{-i\left(\Theta_{0}^{\text {out }}-\Theta_{0}^{\text {in }}\right)} \\
& -R_{21} e^{i\left(\Theta_{0}^{\text {out }}-\Theta_{0}^{\text {in }}\right)}
\end{aligned}
$$



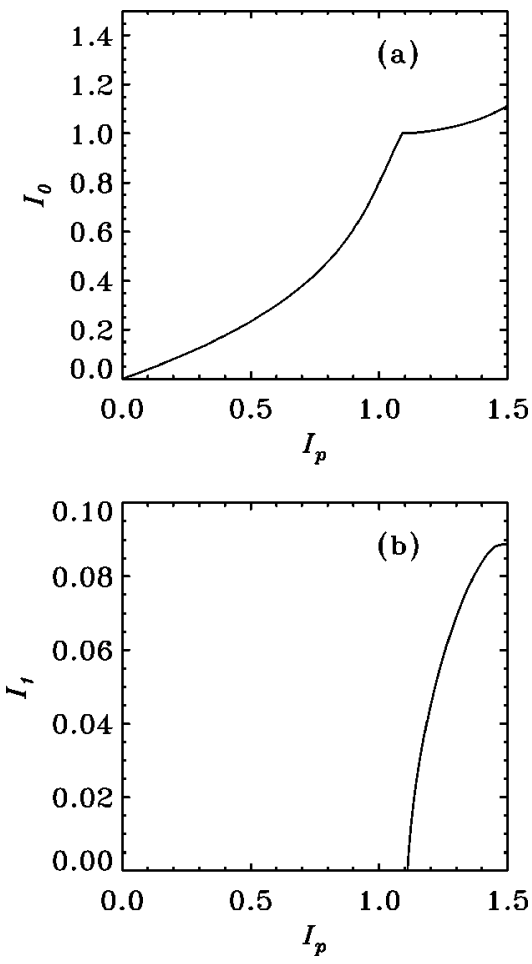

FIG. 2. Steady state in the self-focusing case. (a) Intensity of $I_{0}$ as a function of the driving field intensity $I_{p}$. (b) Intensity of $I_{1}$ $=g\left|a_{1}^{s}\right|^{2}$ as a function of $I_{p}$. Parameters: $\theta_{0}=1.3, \theta_{1}=2$.

$$
\begin{aligned}
\left\langle\delta X_{0}^{\text {in }} \delta X_{+}^{\text {out }}\right\rangle_{\omega}= & {\left[\left(R_{41}+R_{31}+R_{61}+R_{51}\right) e^{-i \Theta_{0}^{\text {in }}}\right.} \\
& \left.-\left(R_{42}+R_{32}+R_{62}+R_{52}\right) e^{i \Theta_{0}^{\text {in }}}\right] / \sqrt{2} .
\end{aligned}
$$

$\delta X_{0}^{\text {in }}=\delta a_{0} e^{-i \Theta_{0}^{\text {in }}}+\delta a_{0}^{\dagger} e^{i \Theta_{0}^{\text {in }}}$ denotes the fluctuations of the coherent input pump in the quadrature component corresponding to the input intensity; since $a_{0}^{\text {in }}=\alpha_{\mathrm{I}} e^{-i \phi_{0}}, \Theta_{0}^{\text {in }}=$ $-\phi_{0}$.

Since the input beam is in a coherent state, the fluctuations correspond to the shot-noise level and $\left\langle\delta X_{0}^{\mathrm{in}} \delta X_{0}^{\mathrm{in}}\right\rangle_{\omega}$ $=1$. The condition for achieving QND performances is $C_{s}$ $+C_{m}>1$.

\section{RESULTS}

We present first the results concerning the self-focusing case $(\eta=1)$. From the stability analysis of the continuous semiclassical model [26] we know that the first modes that become unstable are characterized by a critical wave number $k_{c}=\sqrt{\left(2-\theta_{0}\right) / a}$. So, from Eq. (19) we obtain that the detuning of the critical modes is $\theta_{1}=2$. For $\eta=1$ the Hamiltonians (18)-(23) are independent of $\alpha$ so that in the selffocusing case we are left with only one free parameter $\theta_{0}$, which can be adjusted to optimize the results. However, its value cannot exceed 41/30 for the pattern formation bifurcation to be supercritical [26], which guarantees that the amplitude of the pattern modes is small close to threshold.

We show an example of the three-mode steady-state solution in Fig. 2 for $\theta_{0}=1.3$. As shown in the figure, the instability threshold for pattern formation takes place at $I_{p}^{\text {th }}$
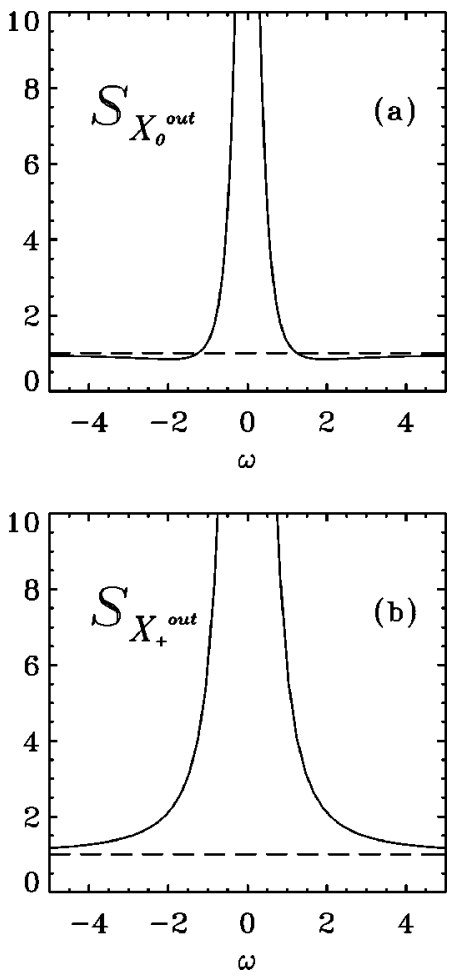

FIG. 3. Squeezing spectra in the self-focusing case. $X$ axis: scaled frequency $\omega . \quad Y$ axis: (a) squeezing spectrum $S_{X_{0}^{\text {out }}},($ b) squeezing spectrum $S_{X_{+}^{\text {out }}}$. Parameters: $\theta_{0}=1.3, \theta_{1}=2, I_{1}=0.04$.

$=g \alpha_{I \mathrm{th}}{ }^{2}=1.1$. We take a value for the pump $I_{p}=g \alpha_{I}^{2}=1.3$ which is close to the threshold and for which $I_{1}=0.04$. In Fig. 3 we plot the squeezing spectra $S_{X_{0}^{\text {out }}}$ and $S_{X_{+}^{\text {out }}}$ for these values of $\theta_{0}$ and $I_{1}$. As shown in the figure, the squeezing spectrum $S_{X_{+}^{\text {out }}}$ never goes below the shot-noise level $\left(S_{X_{+}^{\text {out }}}\right.$ $=1$ ), so there is no squeezing in the fluctuations of the sum of the tilted modes. Fluctuations in the the homogeneous mode go slightly below shot-noise level for frequencies $|\omega|$ $>1.2$. Note that as we have scaled the time with $\kappa, \omega$ is a dimensionless frequency. The actual frequency would be $\kappa \omega$.

Figure 4 shows the result for the correlation between the outgoing signal and the outgoing meter $V_{s \mid m}\left[X_{0}^{\text {out }} \mid X_{+}^{\text {out }}\right]$, between the incoming and the outgoing signal $C_{s}$, and between the incoming signal and outgoing meter $C_{m}$. We find that $V_{s \mid m}\left[X_{0}^{\text {out }} \mid X_{+}^{\text {out }}\right]$ lies in the QND domain for frequencies $|\omega|$ $>0.2$, although it never reaches values smaller than 0.75 . Still the fact that it is below shot-noise level indicates that a self-focusing Kerr medium can be used for quantum state preparation of the homogeneous output mode by acting on the quantum state of the meter modes. On the other hand, the condition $C_{s}+C_{m}>1$ is not fulfilled, indicating a poor correlation between the incoming and outgoing signal and meter. This fact precludes the possibility of a QND measurement of the fluctuations of the input pump beam by using the fluctuations of the output tilted modes as meter. Similar or worse results are obtained for other values of the detuning $\theta_{0}$ within the range $\theta_{0}<41 / 30$.

The results for the correlation $V_{s \mid m}$ improve significantly if we consider the correlation between $X_{+}^{\text {out }}$ and the quadrature component $X_{0}$ instead of $X_{0}^{\text {out }}$, where $X_{0}=a_{0}+a_{0}^{\dagger}$ cor- 

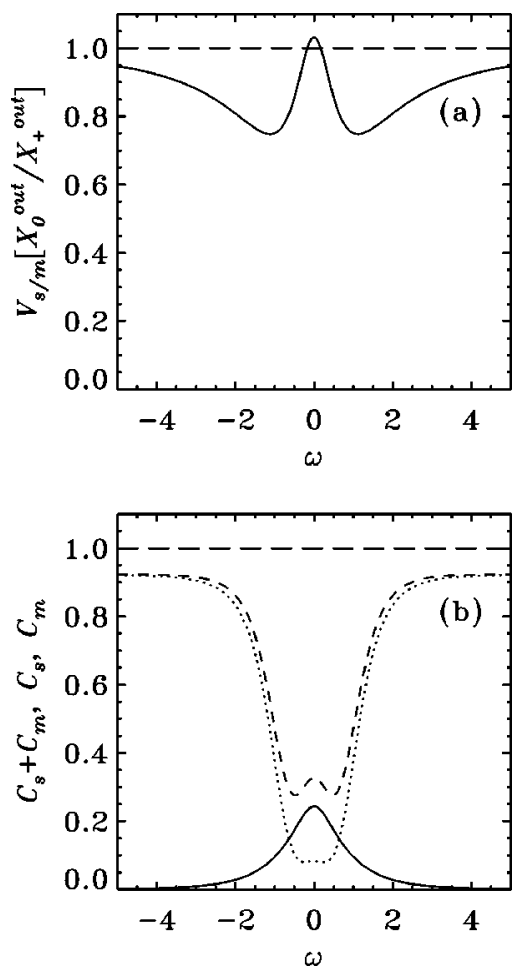

FIG. 4. Self-focusing case. (a) Conditional variance $V_{s \mid m}\left[X_{0}^{\text {out }} \mid X_{+}^{\text {out }}\right]$. (b) Coefficients $C_{s}$ (dotted line), $C_{m}$ (solid line), and $C_{s}+C_{m}$ (dashed line). Same parameters as in Fig. 3.

responds to the amplitude quadrature inside the cavity. $X_{0}$ can be described as a linear combination of the amplitude and phase quadratures of the field outside the cavity. Therefore it can be observed using a local oscillator instead of performing a direct intensity detection. The conditional variance $V_{s \mid m}\left[X_{0} \mid X_{+}^{\text {out }}\right]$ exhibits, for zero frequency, a minimum much more pronounced than that of $V_{s \mid m}\left[X_{0}^{\text {out }} \mid X_{+}^{\text {out }}\right]$, with values smaller than 0.3 for the same values of the detuning and pump used before.

For the self-defocusing vector case $(\eta=-1)$, the linear stability analysis of the semiclassical equations $[27,28]$ shows that the first transverse modes that become unstable have a wave number $k_{c}=\sqrt{\left[\theta_{0}-\alpha /(1-\alpha)\right] / a}$, and $\theta_{1}$ $=\alpha /(1-\alpha)$. In this case we therefore have two free parameters, $\alpha$ and $\theta_{0}$. We need to keep $\theta_{0}<\sqrt{3}$, to avoid bistability of the homogeneous solution, and $\theta_{0}>\theta_{1}$, in order to have a nonzero critical wave number. We first consider the case $\alpha=1 / 4$, which is a typical value for a liquid Kerr medium, so that $\beta=7 / 4$ and $\theta_{1}=1 / 3$. Figure 5 displays the steady-state value for $I_{1}$ and $I_{0}$ as functions of the input field $I_{p}$ for $\theta_{0}=1.7$. In this case the threshold for pattern formation is located at $I_{p}^{\text {th }}=1.51$. Figure 6 shows the squeezing spectra close to threshold, $I_{p}=1.76$, so that $I_{1}=0.06$. The spectrum of fluctuations for the sum of the $y$-polarized tilted modes is very similar to the one obtained in the self-focusing case and it does not go below the shot-noise level. The spectrum of fluctuations for the output homogeneous $x$-polarized mode does in fact go below shot-noise level for frequencies $|\omega|>0.8$, reaching a minimum value of $S_{X_{0}^{\text {out }}}=0.4$.

Figure 7 shows the correlations $V_{s \mid m}\left[X_{0}^{\text {out }} \mid X_{+}^{\text {out }}\right], C_{s}$, and $C_{m}$ for the self-defocusing case. Particularly interesting is that despite the fact that there is no squeezing for $X_{0}$ at $\omega$
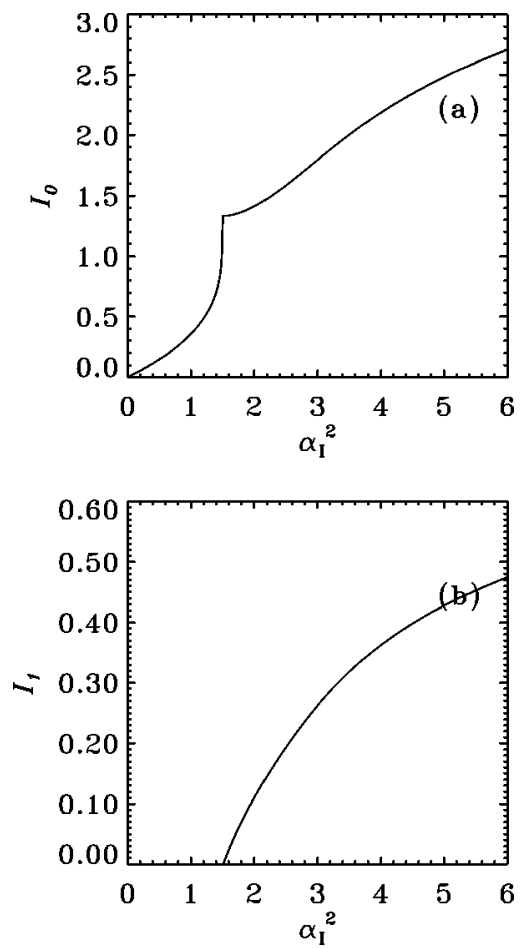

FIG. 5. Steady state in the self-defocusing case. (a) Intensity of $I_{0}$ as a function of the driving field intensity $I_{p}$. (b) Intensity of $I_{1}=g\left|a_{1}^{s}\right|^{2}$ as a function of $I_{p}$. Parameters: $\theta_{0}=1.7, \theta_{1}=1 / 3, \alpha$ $=0.25$.
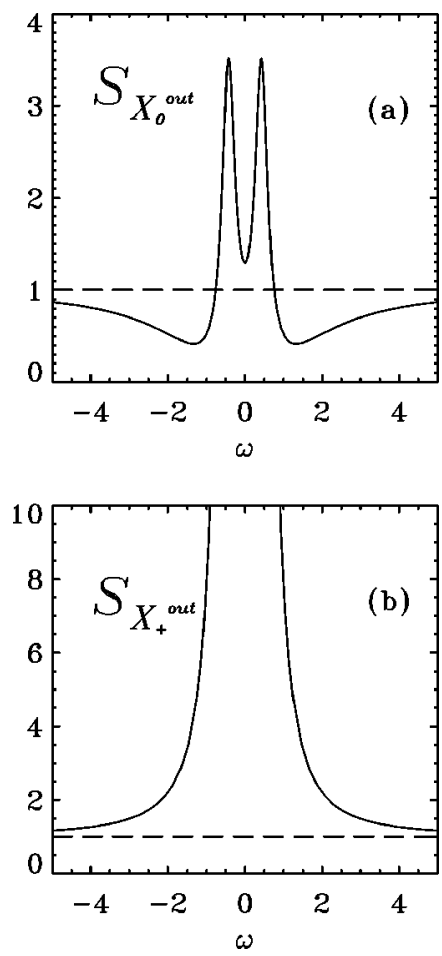

FIG. 6. Squeezing spectra in the self-defocusing case. $X$ axis: scaled frequency $\omega / \kappa . \quad Y$ axis: (a) squeezing spectrum $S_{X_{0}^{\text {out }}}$, (b) squeezing spectrum $S_{X_{+}^{\text {out. }}}$ Parameters: $\theta_{0}=1.7, \theta_{1}=1 / 3, \alpha$ $=0.25, I_{1}=0.06$. 

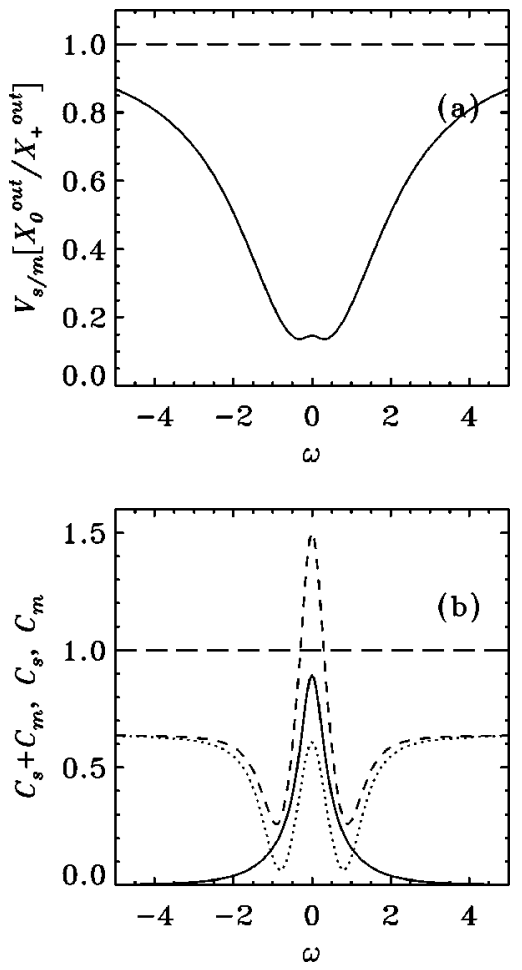

FIG. 7. Self-defocusing case. (a) Conditional variance $V_{s \mid m}\left[X_{0}^{\text {out }} \mid X_{+}^{\text {out }}\right]$. (b) Coefficients $C_{s}$ (dotted line), $C_{m}$ (solid line), and $C_{s}+C_{m}$ (dashed line). Same parameters as in Fig. 6

$=0$, the correlation between the outgoing signal and the outgoing meter is clearly below the shot-noise level. These strong correlations are the quantum counterpart of the ones found classically in the far field between the fluctuations in the $x$-polarized pump beam and the fluctuations in the $y$-polarized modes with wave vectors $\pm \vec{k}_{c}$ [29]. The result for $V_{s \mid m}\left[X_{0}^{\text {out }} \mid X_{+}^{\text {out }}\right]$ implies that we can use a vectorial selfdefocusing Kerr medium to prepare a state of the homogeneous output mode with known fluctuations. Compared with the self-focusing case, the advantage is that now the correlations are much stronger $\left(V_{s \mid m}\left[X_{0}^{\text {out }} \mid X_{+}^{\text {out }}\right]\right.$ reaches a minimum value of 0.13 ). What is more important is that now the coefficients $C_{s}$ and $C_{m}$ satisfy the condition $C_{s}+C_{m}>1$ for the range of frequencies $|\omega|<0.4$. In this range of frequencies a QND measurement of the $x$-polarized input fluctuations can be done using the $y$-polarized pattern modes as meter.

Decreasing the value of the detuning $\theta_{0}$, the results for $V_{s \mid m}\left[X_{0}^{\text {out }} \mid X_{+}^{\text {out }}\right], C_{s}$, and $C_{m}$ become worse. However, for pumping levels close to the pattern formation instability threshold, the conditions for a QND measurement are fulfilled in the range $\sqrt{3} \geqslant \theta_{0}>1.6$. On the other hand, the results for the correlations $V, C_{s}$, and $C_{m}$ can be improved if we consider different values of the nonlinear coefficient $\alpha$. For example, for $\alpha=0.15$ and detuning $\theta_{0}=1.7$, the pattern formation instability threshold takes place at $I_{p}^{\text {th }}=1.50$. For pump intensity $I_{p}=1.73$, so that $I_{1}=0.06$, we have almost perfect QND conditions, that is, $V_{s \mid m}\left[X_{0}^{\text {out }} \mid X_{+}^{\text {out }}\right]$ is close to 0 and $C_{s}+C_{m}$ close to 2 at $\omega=0$ (see Fig. 8).

\section{SUMMARY AND CONCLUSIONS}

We have studied the quantum correlation between the fluctuations of the pump and the fluctuations of the trans-
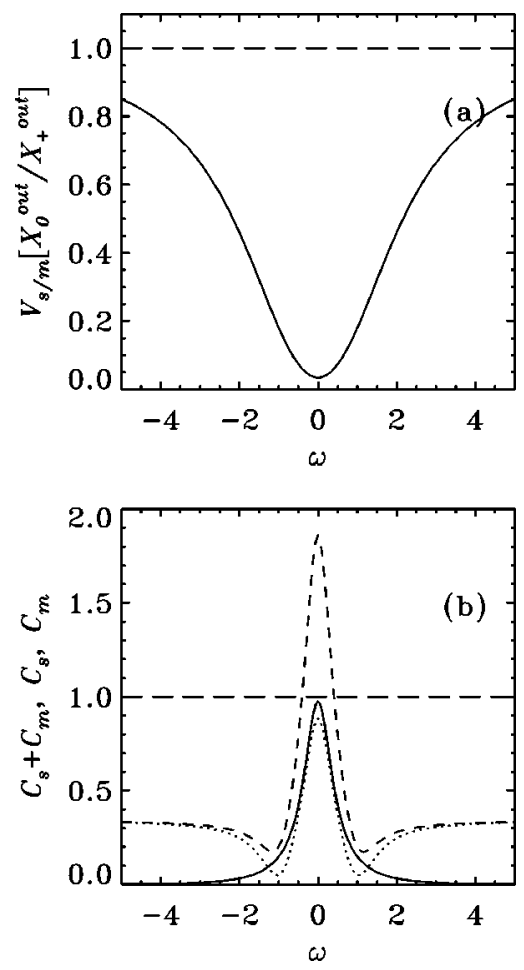

FIG. 8. Self-defocusing case. (a) Conditional variance $V_{s \mid m}\left[X_{0}^{\text {out }} \mid X_{+}^{\text {out }}\right]$. (b) Coefficients $C_{s}$ (dotted line), $C_{m}$ (solid line), and $C_{s}+C_{m}$ (dashed line). Same parameters as in Fig. 6 except the nonlinear coefficient $\alpha$. Here we take $\alpha=0.15$.

verse modes above the threshold for spatial instability in a Kerr medium, including also the polarization degree of freedom. We have considered two cases in which a stripe pattern is formed when the system is pumped with a linearly $x$-polarized input field. In the first case we consider a transverse one dimensional self-focusing Kerr medium and the stripe pattern is also linearly $x$ polarized. In this case the polarization degree of freedom plays no role (scalar case). In the second case (vectorial case) we consider a transverse bidimensional self-defocusing Kerr medium and the stripe pattern is orthogonally polarized to the pump. In both cases our theoretical description is reduced to a three-mode model: a homogeneous mode corresponding to the pump and two modes associated with the transverse pattern.

While in both cases we found anticorrelations between the quantum fluctuations of the pump intensity and the sum of the intensities of the stripe pattern, they turn out to be much stronger in the vectorial case. We have analyzed the possibility of using the system as a QND device taking the beams associated with transverse stripe pattern as meter beams to measure the fluctuations of the pump beam. We have calculated the three correlation coefficients that measure correlations between incoming and outgoing signal (pump), between incoming signal and outgoing meter, and between outgoing signal and outgoing meter. We have shown that all the conditions for a QND measurement are satisfied in the vectorial case within a range of parameters. The best results were obtained for detunings close to bistability.

Our results confirm the possibility of a QND measurement in a quantum structure [37] where the cause of the pattern formation is a polarization instability and where quantum correlations between pump and meter can be physi- 
cally described as polarization anticorrelations.

For the range of parameters that we have explored, the quantum nature of the anticorrelation between signal and meter is also manifested for the scalar case. This can be used for quantum state preparation of the homogeneous component of the output field by acting on the meter modes. However, the fact that the QND conditions for correlations between the incoming and outgoing fluctuations of the signal and between the incoming signal fluctuations and outgoing meter fluctuations are not satisfied precludes the possibility of a QND measurement in this case.

\section{ACKNOWLEDGMENTS}

This work was supported by the European Comission through the TMR network QSTRUCT (Project No. ERB FMRX-CT96-0077). Financial support from DGICYT (Spain) under Project No. PB94-1167 is also acknowledged. M.H. wants to acknowledge financial support from the FOMEC under Project No. 290, Departamento de Física FCEyN, Universidad Nacional de Mar del Plata, Argentina.

\section{APPENDIX}

Since Eqs. (32) and (33) are similar to Eqs. (37) and (38), we obtain similar drift and diffusion matrices, and we can present them in a unified form as follows (we only show the nonzero components of $A$ and $D$ ):

$$
\begin{gathered}
A_{1,1}=A_{2,2}^{*}=1-i\left(2 \eta I_{0}+4 \eta I_{1}-\eta \theta_{0}-2 u I_{1}+4 I_{1}\right), \\
A_{1,2}=A_{2,1}^{*}=\frac{2 I_{1}-i \eta\left(I_{0}^{2}+2 \theta_{1} I_{1}-2 u I_{0} I_{1}-6 I_{1}^{2}\right)}{I_{0}}
\end{gathered}
$$

$$
\begin{gathered}
A_{1,3}=A_{1,5}=A_{2,4}^{*}=A_{2,6}^{*}=-A_{3,1}^{*}=-A_{5,1}^{*}=-A_{4,2}=-A_{6,2} \\
=\sqrt{\frac{I_{1}}{I_{0}}}\left[2+i \eta\left(u I_{0}-2 \theta_{1}+6 I_{1}\right)\right], \\
A_{1,4}=A_{1,6}=-A_{4,1}=-A_{6,1}=-A_{2,3}=-A_{2,5}=A_{3,2}=A_{5,2} \\
=-i \eta u \sqrt{I_{0} I_{1}}, \\
A_{3,3}=A_{5,5}=A_{4,4}^{*}=A_{6,6}^{*} \\
=1-i\left(2 \eta I_{0}+4 \eta I_{1}-\eta \theta_{1}-u I_{0}+2 I_{0}\right), \\
A_{3,4}=A_{5,6}=-A_{4,3}=-A_{6,5}=-i \eta I_{1}, \\
A_{3,5}=A_{5,3}=-A_{4,6}=-A_{6,4}=-2 i \eta I_{1}, \\
A_{3,6}=A_{4,5}^{*}=A_{5,4}=A_{6,3}^{*}=-1+i \eta\left(u I_{0}-\theta_{1}+I_{1}\right), \\
D_{1,1}=D_{2,2}^{*}=\frac{-2 I_{1}+i \eta\left(I_{0}^{2}+2 \theta_{1} I_{1}-2 u I_{0} I_{1}-6 I_{1}^{2}\right)}{I_{0}}, \\
D_{1,3}=D_{1,5}=D_{3,1}=D_{5,1}=-D_{2,4}=-D_{2,6}=-D_{4,2}=D_{4,6}^{*}=D_{6,4}^{*}=1-i \eta\left(u I_{0}-\theta_{1}+I_{1}\right), \\
=-D_{6,2}=i \eta u \sqrt{I_{0} I_{1}}, \\
D_{3,3}=D_{4,4}^{*}=D_{5,5}=D_{6,6}^{*}=i \eta I_{1}, \\
\text { A } 2)
\end{gathered}
$$

where the constant $u$ takes different values depending on the case: for self-focusing it is $u=2$ and for self-defocusing $u$ $=\alpha$.
[1] L. A. Lugiato, Chaos Solitons Fractals 4, 1251 (1994), and references quoted therein; L. A. Lugiato, M. Brambilla, and A. Gatti, in Advances in Atomic Molecular and Optical Physics, edited by B. Bederson and H. Walther (Academic Press, New York, 1998), Vol. 40, p. 229.

[2] L. A. Lugiato, A. Gatti, and H. Wiedemann, in Quantum Fluctuations, edited by S. Reynaud, E. Giacobino, and J. Zinn Justin (Elsevier-North-Holland, Amsterdam, 1997).

[3] L. A. Lugiato and F. Castelli, Phys. Rev. Lett. 68, 3284 (1992).

[4] G. Grynberg and L. A. Lugiato, Opt. Commun. 101, 69 (1993).

[5] M. Santagiustina, P. Colet, M. San Miguel, and D. Walgraef, Phys. Rev. Lett. 79, 3633 (1997).

[6] L. A. Lugiato and A. Gatti, Phys. Rev. Lett. 70, 3868 (1993).

[7] A. Gatti and L. A. Lugiato, Phys. Rev. A 52, 1675 (1995).

[8] L. A. Lugiato and I. Marzoli, Phys. Rev. A 52, 4886 (1995).

[9] M. Kolobov and L. A. Lugiato, Phys. Rev. A 52, 4930 (1995).

[10] L. A. Lugiato and G. Grynberg, Europhys. Lett. 29, 675 (1995)

[11] I. Marzoli, A. Gatti, and L. A. Lugiato, Phys. Rev. Lett. 78, 2092 (1997).

[12] A. Gatti, H. Wiedemann, L. A. Lugiato, I. Marzoli, G. L. Oppo, and S. M. Barnett, Phys. Rev. A 56, 877 (1997).

[13] L. A. Lugiato, A. Gatti, H. Ritsch, I. Marzoli, and G. L. Oppo, J. Mod. Opt. 44, 1899 (1997).
[14] L. A. Lugiato and Ph. Grangier, J. Opt. Soc. Am. B 14, 225 (1997).

[15] A. Gatti, L. A. Lugiato, G. L. Oppo, R. Martin, P. Di Trapani, and A. Berzanskis, Opt. Express 1, 21 (1997).

[16] F. Castelli and L. A. Lugiato, J. Mod. Opt. 44, 765 (1997).

[17] M. Santagiustina, P. Colet, M. San Miguel, and D. Walgraef, Phys. Rev. E 58, 3843 (1998).

[18] M. I. Kolobov and I. V. Sokolov, Zh. Éksp. Teor. Fiz. 96, 1945 (1989) [Sov. Phys. JETP 69, 1097 (1989)].

[19] M. I. Kolobov and I. V. Sokolov, Phys. Lett. A 140, 101 (1989).

[20] M. I. Kolobov and I. V. Sokolov, Europhys. Lett. 15, 271 (1991).

[21] M. I. Kolobov, Phys. Rev. A 44, 1986 (1991).

[22] M. I. Kolobov and P. Kumar, Opt. Lett. 18, 849 (1993).

[23] B. M. Jost, A. V. Sergienko, A. F. Abouraddy, B. E. A. Saleh, and M. C. Feich, Opt. Exp. 3, 81 (1998).

[24] C. Sibilia, V. Schiavone, M. Bertolotti, R. Horak, and I. Perina, J. Opt. Soc. Am. B 11, 2175 (1994).

[25] E. M. Nagasako, R. W. Boyd, and G. S. Agarwal, Phys. Rev. A 55, 1412 (1997).

[26] L. A. Lugiato and L. Lefever, Phys. Rev. Lett. 58, 2209 (1987). 
[27] J. B. Geddes, J. V. Moloney, E. M. Wright, and W. J. Firth, Opt. Commun. 111, 623 (1994).

[28] M. Hoyuelos, P. Colet, M. San Miguel, and D. Walgraef, Phys. Rev. E 58, 2992 (1998).

[29] M. Hoyuelos, P. Colet, and M. San Miguel, Phys. Rev. E 58, 74 (1988).

[30] Ph. Grangier, Phys. Rep. 219, 121 (1992).

[31] M. J. Holland, M. Collett, D. F. Walls, and M. D. Levenson, Phys. Rev. A 42, 2995 (1990).

[32] Notice that the correspondence with the notation used in $[27,28]$ is $\alpha=A$ and $\beta=A+B$.

[33] This relation comes from the fact that for an isotropic Kerr medium the elements of the susceptibility tensor satisfy $\chi_{1111}$ $=\chi_{1122}+\chi_{1212}+\chi_{1221}$, and the parameters $\alpha$ and $\beta$ are $\alpha$ $=\left(\chi_{1122}+\chi_{1212}\right) / \chi_{1111}$ and $\beta=\left(\chi_{1122}+\chi_{1212}+2 \chi_{1221}\right) / \chi_{1111}$. See, for example, R. W. Boyd, Nonlinear Optics (Academic Press, Boston, 1992).

[34] Notice that in Ref. [3] $g$ was defined as negative whereas it is here taken as positive.

[35] D. F. Walls and G. J. Milburn, Quantum Optics (SpringerVerlag, Heidelberg, 1994).

[36] A. Sinatra, J. F. Roch, K. Vigneron, Ph. Grelu, J. Ph. Poizat, Kaige Wang, and P. Grangier, Phys. Rev. A 57, 2980 (1998).

[37] Optics Express 3 (2), 59 (1998) (http://epubs.osa.org/ opticsexpress), focus issue on Quantum Structures in Nonlinear Optics and Atomic Physics, edited by G. L. Oppo and L. A. Lugiato. 